\title{
Lost of Bosonic Coherence Due to the Fermionic Presence Under the Synthetic Magnetic Field
}

\begin{abstract}
T.P. POLAK
Faculty of Physics, Adam Mickiewicz University of Poznań, Umultowska 85, 61-614 Poznań, Poland

We have studied effects of inter-species interaction of the bosons in a Bose-Fermi mixture over a large regime of particle numbers under the synthetic magnetic field. Analytically derived formulas for the density of states for several values of the magnetic fields $f=1 / 2,1 / 3,1 / 4,1 / 6,1 / 8$ and $3 / 8$ allows us to calculate, with a very good accuracy, the effective interaction between bosons. The presence of the Hofstadter butterfly spectrum and fermionic species induces alternating sign potential between neutral bosonic atoms. Consequently bosons can attract or repulse each other whether sign of it strongly depends on the strength of the magnetic field applied to the sample.
\end{abstract}

PACS: 05.30.Jp, 03.75.Lm, 03.75.Nt

Trapping and cooling Bose-Fermi mixtures of dilute quantum gases has opened a wide area of research in atomic physics. The interactions between bosonic and fermionic species interconnect two systems of fundamentally different quantum statistics. The charge neutrality of atoms prevents direct application of the Lorentz force with a magnetic field. Recently, the observation of a great variety of fundamental phenomena i.e. quantumHall effects become possible owing to the advancements of various experimental techniques [1-3]. An equivalent effect can be provided by the Coriolis force in a rotating atomic gas. In a frame of reference rotating about the $z$-axis with angular velocity $\Omega$ the kinetic term in Hamiltonian is equivalent to that of a particle of charge $Q$ experiencing a magnetic field $B$ with $Q B=2 \mathrm{~m} \Omega$, where $m$ is the mass of the particle [4]. The above setting comes with limitations because a large magnetic fields $f \equiv m a^{2} \Omega / \pi \hbar$ (angular momentum) are required to make possible the study of poorly explored bosonic states in case when $f \equiv p / q,(p$ and $q$ is the ratio of atom number to the number of flux quanta respectively) is a rational number.

Current experiments [5-9] on trapped mixtures of the atomic Bose-Fermi (BF) and Bose-Bose gases show that the presence of a relevant fraction of one modifies the quantum phase transition occurring in the other inducing a significant loss of coherence. These observations are supported by a theoretical description that includes the multiband virtual transitions [10], different masses of strongly interacting particles [11] and numerical calculations [12]. The density-density (DD) interaction between different species can be repulsive or attractive and is produced by changes of one species density that induce a modulation of another. Therefore the dynamics underlying the phase transitions in the BF mixtures is produced by the feedback of the density perturbation and a shift of the inter-bosonic potential occurs, that changes the original interaction between them [13] providing various novel phases [14].

In the present paper, motivated by recent experiments done by Lin's group et al. [15] which engineered a Hamiltonian with a spatially dependent vector potential $\boldsymbol{A}$ thus successfully producing $\boldsymbol{B}=\nabla \times \boldsymbol{A}$, we calculated the form of the effective inter-bosonic potential when a synthetic magnetic field (SMF) is applied to neutral gaseous Bose-Fermi mixtures. We predict that the fermionmediated effective interaction between bosons has a complicated pattern of the frequency dependent magnitude. Moreover, the SMF renders the inter-bosonic potential oscillatory with sign change, thus switching it between repulsive and attractive. As a consequence the resonances appear and BF mixture that enters the quantum-Hall regime displays surprisingly complex dynamics unreachable in conventional solid state physics. We expect that our theoretical results open up the experimental studies [16] of the re-normalized interaction energies in stable many-body phases with strong correlations and their dynamical properties.

Restricting our analysis to the lowest energy band of a square optical lattice in synthetic magnetic field, the Bose-Fermi quantum gaseous mixture can be modeled via the following Hamiltonian [17]:

$$
\begin{aligned}
\hat{\mathcal{H}} & =\frac{U_{b}}{2} \sum_{i} \hat{n}_{b i}\left(\hat{n}_{b i}-1\right)-\sum_{\langle i, j\rangle} t_{b i j} \mathrm{e}^{\frac{2 \pi \mathrm{i}}{\Phi_{0}} \int_{\boldsymbol{r}_{j}}^{\boldsymbol{r}_{i}} \boldsymbol{A} \cdot \mathrm{d} \boldsymbol{l}} \hat{b}_{i}^{\dagger} \hat{b}_{j} \\
& -\mu_{b} \sum_{i} \hat{n}_{b i}-\sum_{\langle i, j\rangle} t_{f i j} \mathrm{e}^{\frac{2 \pi i}{\Phi_{0}} \int_{\boldsymbol{r}_{j}}^{\boldsymbol{r}_{i}} \boldsymbol{A} \cdot \mathrm{d} \boldsymbol{l} \hat{c}_{i}^{\dagger}} \hat{c}_{j}-\mu_{f} \sum_{i} \hat{n}_{f i} \\
& +U_{b f} \sum_{i} \hat{n}_{b i} \hat{n}_{f i},
\end{aligned}
$$

where $\hat{b}_{i}^{\dagger}\left(\hat{c}_{i}^{\dagger}\right)$ and $\hat{b}_{j}\left(\hat{c}_{j}\right)$ stand for the bosonic (fermionic) creation and annihilation operators; $\hat{n}_{b i}=\hat{b}_{i}^{\dagger} \hat{b}_{i}\left(\hat{n}_{f i}=\right.$ $\left.\hat{c}_{i}^{\dagger} \hat{c}_{i}\right)$ measures the corresponding boson (fermion) number on the site $i, U_{b}>0$ is the on-site repulsion and 
$\mu_{b}\left(\mu_{f}\right)$ stands for the chemical potential for bosons (fermions). The DD interaction between the bosonic and non-interacting, spin-polarized (collisions in the $s$ wave channel are forbidden by their statistics), fermionic atoms is denoted by $U_{b f}$ and depends, on boson to fermion mass ratio $m_{b} / m_{f}$. Here, $\langle i, j\rangle$ identifies summation over the nearest-neighbor sites. Furthermore, $t_{b}\left(t_{f}\right)$ sets the kinetic energy scale for bosons (fermions). We assume that an optical lattice created by the counterpropagating laser beams is deep enough and we can restrict ourselves to the lowest Bloch bands. The corresponding experimental parameters can be estimated by following relations:

$$
\begin{aligned}
t_{x} & \simeq \frac{4}{\sqrt{\pi}} E_{r}^{x}\left(\frac{V_{0}}{E_{r}^{x}}\right)^{3 / 4} \exp \left(-2 \frac{V_{0}}{E_{r}^{x}}\right), \\
U_{x} & \simeq \sqrt{\frac{8}{\pi}} k a_{x} E_{r}^{x}\left(\frac{V_{0}}{E_{r}^{x}}\right)^{3 / 4},
\end{aligned}
$$

(subscript $x=\{b, f\}$ means $b$ bosons and $f$ fermions respectively) where boson-boson $a_{b}$, fermion-fermion $a_{f}$ and boson-fermion $a_{b f}$

$$
U_{b f} \simeq \sqrt{\frac{8}{\pi}} k a_{b f} E_{r}^{b}\left(\frac{V_{0}}{E_{r}^{b}}\right)^{3 / 4} \frac{1+\frac{m_{b}}{m_{f}}}{\left(1+\sqrt{\frac{m_{b}}{m_{f}}}\right)^{3 / 2}},
$$

scattering lengths can be continuously tune in the experiments [5-8] inducing attractive or repulsive interaction between species. The $k=2 \pi / \lambda$ is the wavelength of the laser and $E_{r}^{x}=\hbar^{2} k^{2} / 2 m_{x}$ is the recoil energy and $m_{x}$ is the atomic mass. The phase shift on each site is determined by the vector potential $\boldsymbol{A}(\boldsymbol{r})$ and can be controlled experimentally [15] and can be interpreted as AharonovBohm phases. The magnetic field is introduced in the theory by the density of states (DOS). There are significant difficulties in obtaining and analyzing and analyze the solutions of the above analytically for every value of $f$. Only a few closed formulas for DOS are accessible [18] and consequently not every applied magnetic field can be described theoretically. Of special interest are the values of the angular momentum which correspond to rational numbers of $f=1 / 2,1 / 3,1 / 4, \ldots$ Since all properties of the Hamiltonian (1) are invariant under $f \rightarrow-f$ and also under $f \rightarrow f+1$, it is sufficient to consider $f$ in the range $0<f<1 / 2$ that can be reached experimentally by applying Raman lasers to the gas in order to realize a Berry's phase for a moving particle. This allows us the detailed analysis of the dynamical response function which have been found to play a crucial role in complex systems. The partition function of bosonic and fermionic mixtures is written in the form $\mathcal{Z}=\int[\mathcal{D} \bar{b} \mathcal{D} b \mathcal{D} \bar{c} \mathcal{D} c] \mathrm{e}^{-\mathcal{S}[b, c]}$ with action given by

$$
\mathcal{S}=\mathcal{S}_{b}+\mathcal{S}_{c}+\int_{0}^{\beta} \mathrm{d} \tau \mathcal{H}(\tau),
$$

where $\mathcal{S}_{b}=\sum_{i} \int_{0}^{\beta} \mathrm{d} \tau \bar{b}_{i} \frac{\partial}{\partial \tau} b_{i}$ and $\mathcal{S}_{c}=\sum_{i} \int_{0}^{\beta} \mathrm{d} \tau \bar{c}_{i} \frac{\partial}{\partial \tau} c_{i}$. Using the bosonic (fermionic) path integral over the complex fields depending on the "imaginary time" $0 \leq \tau \leq$ $\beta \equiv 1 / k_{\mathrm{B}} T$ with $T$ being the temperature we can easily integrate over the fermionic fields [11] because spins are frozen due to influence of the magnetic trap and there is no direct interaction between fermions. After that, we obtain the partition function in the form

$$
\mathcal{Z}=\int[\mathcal{D} \bar{b} \mathcal{D} b \mathcal{D} \bar{c} \mathcal{D} c] \mathrm{e}^{-\mathcal{S}_{b}\left[b, n_{b}\right]} \mathrm{e}^{-\operatorname{Tr} \ln G_{c}}
$$

The trace of the two-point correlation function for non-interacting fermions $G_{c}$, after exploiting FourierMatsubara transform reads:

$$
\operatorname{Tr} \ln G_{c}=-\frac{U_{b f}^{2}}{2} \sum_{\boldsymbol{k}, \ell} \Lambda_{\boldsymbol{k}}\left(\omega_{\ell}\right) \chi_{\boldsymbol{k}}\left(\mathrm{i} \nu_{\ell}\right) \Lambda_{-\boldsymbol{k}}\left(-\omega_{\ell}\right),
$$

where $\omega_{\ell}=2 \pi \ell / \beta\left(\nu_{\ell}=\pi(2 \ell+1) / \beta\right)$ with $\ell=$ $0, \pm 1, \pm 2, \ldots$ are the Bose(Fermi)-Matsubara frequencies respecting periodic (anti-periodic) boundary conditions of the bosonic (fermionic) field operator with $\Lambda_{k}\left(\omega_{\ell}\right)=$ $\bar{b}_{\boldsymbol{k}}\left(\omega_{\ell}\right) b_{\boldsymbol{k}}\left(\omega_{\ell}\right)$ and

$$
\chi_{\boldsymbol{k}}\left(\mathrm{i} \nu_{\ell}\right)=\sum_{\boldsymbol{k}^{\prime}} \frac{n_{\mathrm{F}}\left(t_{f \boldsymbol{k}^{\prime}}^{p / q}\right)-n_{\mathrm{F}}\left(t_{f \boldsymbol{k}^{\prime}+\boldsymbol{k}}^{p / q}\right)}{t_{f \boldsymbol{k}^{\prime}}^{p / q}-t_{f \boldsymbol{k}^{\prime}+\boldsymbol{k}}^{p / q}-\mathrm{i} \nu_{\ell}},
$$

is the Lindhard function - more commonly called the RPA with $n_{\mathrm{F}}(x)$ being the Fermi distribution; $t_{\boldsymbol{k}^{\prime}}^{p / q}$ is the dispersion relation calculated from Harper equation [18]. It correctly predicts a number of properties of the collective phenomena in electron gas such as plasmons [19]. To stay in the local regime we perform $\boldsymbol{k}$ and $\boldsymbol{k}^{\prime}$ integration over the first Brillouin zone and, in the $T \rightarrow 0$ limit, using an analytic continuation, we obtain imaginary part $\chi^{\prime \prime}(\omega)$ of the local dynamic Lindhard function (LDLF). Therefore, the corresponding real part $\chi^{\prime}(\omega)$ can be deduced from the Kramers-Krönig relation. Now, doing the inverse Fourier transform Eq. (7) and using gradient expansion, we obtain quadratic form of the trace with extracted frequency dependence

$$
\operatorname{Tr} \ln G_{c} \rightarrow-\frac{U_{b f}^{2} \chi^{\prime}(\omega)}{2} \sum_{i} \int_{0}^{\beta} \mathrm{d} \tau\left[\bar{b}_{i}(\tau) b_{i}(\tau)\right]^{2} .
$$

The consequence of the difference in masses of bosons and fermions is the fact that the speed of the Bogoliubov sound $v_{b}$ for bosons differs from the first sound $v_{f}$ of the ideal Fermi gas. In typical experimental realizations the acoustic long-wavelength boson and fermion velocities are comparable and both constituents equilibrates similarly. The mentioned different mass ratio has far-reaching consequences, including the possibility of generating the DD oscillations. When we add Eq. (9) to the bosonic part of the action there is a striking resemblance to the one-component Bose-Hubbard action with the original repulsive interaction replaced now by $U_{b} \rightarrow U_{\text {eff }}=U_{b}+U_{b f}^{2} \chi^{\prime}(\omega)$ which is the induced, frequency-dependent, effective inter-bosonic potential. From the above we see the DD correlations between the constituents give rise to additional interaction among bosons, which is robust to repulsive or attractive nature of the inter-species interaction but not to the sign of the LDLF. The interactions caused by the DD corre- 


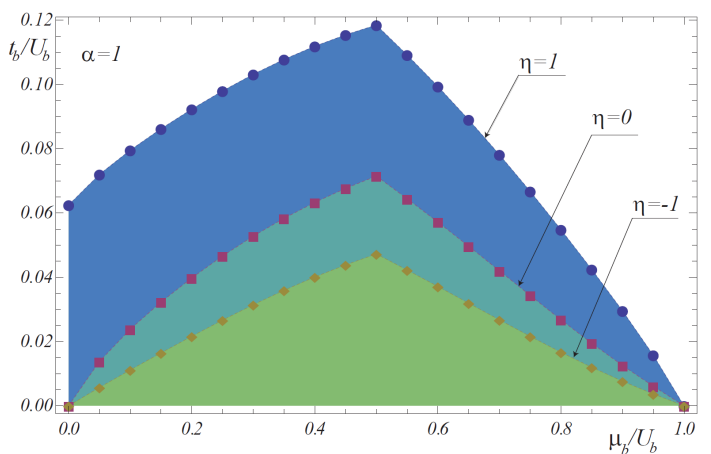

Fig. 1. Real $t_{f} \chi^{\prime \prime}(\omega)$ and imaginary $t_{f} \chi^{\prime}(\omega)$ parts of the local, frequency $\omega / 8 t_{f}$ dependent, density-density response function in the synthetic magnetic field for $f=$ $1 / 3$. Normalization here $t_{f} \equiv t_{f}^{p / q=0}$.

lations may change its sign as a result of the collective excitations see Fig. 1. Such complex behavior of the mixtures emerges in the limit not reachable in conventional systems of condensed matter physics because the very high values of magnetic field are required to acquire the desired range $f \leq 1 / 2$. Experimentally, by using a superlattice potential with more than two non-equivalent sites it is possible to create a lattice with a uniform and non-zero magnetic flux. This system would realize the considered Bose Hubbard model Hamiltonian with the presence of the fractal band structure of the Hofstadter butterfly.

To obtain an equation of state we apply the quantumrotor approach, that successfully casts an essential part of the physics of strongly interacting fermions [20-22] and bosons [23] exclusively, to BF mixtures. We incorporate fully our calculations to the phase fluctuations

$$
b_{i}(\tau)=\left(a_{0}+a_{i}^{\prime}(\tau)\right) \mathrm{e}^{\mathrm{i} \phi_{i}(\tau)},
$$

governed by the gauge $\mathrm{U}(1)$ group and drop corrections to the amplitude $a_{i}^{\prime}(\tau)$ of the order parameter

$$
\Psi_{\mathrm{B}} \equiv\left\langle b_{i}(\tau) \mathrm{e}^{\mathrm{i} \phi_{i}(\tau)}\right\rangle=b_{0} \psi_{\mathrm{B}} .
$$

The non-vanishing value of the $\Psi_{\mathrm{B}}$ signals a bosonic condensation [23]. A phase $\phi_{i}(\tau)$ of the many-body wave function might be arbitrary but correlations among the local phases of its constituents can bring unusual gauge structures. Now, the partition function $\mathcal{Z}=$ $\int[\mathcal{D} \phi] \mathrm{e}^{-\mathcal{S}_{\mathrm{ph}}[\phi]}$, with an effective action can be expressed in phase-only terms

$$
\begin{gathered}
\mathcal{S}_{\mathrm{ph}}[\phi]=\int_{0}^{\beta} \mathrm{d} \tau\left(\sum_{i}\left(\frac{\dot{\phi}_{i}^{2}(\tau)}{2 U_{\mathrm{eff}}}+\frac{\bar{\mu}_{b}}{\mathrm{i} U_{\mathrm{eff}}} \dot{\phi}_{i}(\tau)\right)\right. \\
\left.-\sum_{\langle i, j\rangle} J^{p / q} \mathrm{e}^{\mathrm{i}\left(\phi_{i}(\tau)-\phi_{j}(\tau)\right)}\right) .
\end{gathered}
$$

The phase stiffness coefficient given by $J^{p / q}=$ $t_{b}^{p / q}\left(8 t_{b}^{p / q}+\bar{\mu}_{b}-U_{b f} N_{\mathrm{F}}\right) / U_{b}$ describes the hopping matrix elements renormalized by the amplitude of the order parameter and $N_{\mathrm{F}}$ is the average number of fermions.
The total time derivative Berry phase imaginary term in Eq. (12) is nonzero due to topological phase field configurations with $\phi_{i}(\beta)-\phi_{i}(0)=2 \pi m_{i}\left(m_{i}=0, \pm 1, \pm 2 \ldots\right)$ that results in topological ingredients to the correlator we will see below. Therefore, we concentrate on closed paths in the "imaginary time" $(0, \beta)$ labeled by the integer winding numbers $m_{i}$. The path-integral

$$
\begin{aligned}
\int & {[\mathcal{D} \phi] \ldots } \\
& \equiv \sum_{\left[m_{i}\right]} \int_{0}^{2 \pi}[\mathcal{D} \phi(0)] \int_{\phi_{i}(0)}^{\phi_{i}(\tau)+2 \pi m_{i}}[\mathcal{D} \phi(\tau)] \ldots,
\end{aligned}
$$

includes a summation over $m_{i}$ and in each topological sector the integration goes over the gauge potentials. Therefore, we do not ignore the compactness of the gauge fields. To proceed, we replace the phase degrees of freedom by the uni-modular scalar complex field $\psi$ which satisfies the quantum periodic boundary condition $\psi_{i}(\beta)=\psi_{i}(0)$. This can be conveniently done using the Fadeev-Popov method with Dirac delta functional resolution of unity, where we take $\psi$ as a continuous but constrained (on the average) variable to have the uni-modular value. We introduce

$$
\begin{aligned}
1 & =\int\left[\mathcal{D} \psi \mathcal{D} \psi^{*}\right] \delta\left(\sum_{i}|\psi(\tau)|^{2}-N\right) \\
& \times \delta\left(\psi_{i}-\mathrm{e}^{\mathrm{i} \phi_{i}(\tau)}\right) \delta\left(\psi_{i}^{*}-\mathrm{e}^{-\mathrm{i} \phi_{i}(\tau)}\right),
\end{aligned}
$$

and

$$
\begin{aligned}
& \delta\left(\sum_{i}\left|\psi_{i}(\tau)\right|^{2}-N\right)=\frac{1}{2 \pi \mathrm{i}} \int_{-\mathrm{i} \infty}^{+\mathrm{i} \infty} \mathrm{d} \lambda \\
& \quad \times \exp \left(\int_{0}^{\beta} \mathrm{d} \tau \lambda\left(\sum_{i}\left|\psi_{i}(\tau)\right|^{2}-N\right)\right),
\end{aligned}
$$

where $N$ is the number of lattice sites. Introducing the Lagrange multiplier $\lambda$, which adds the quadratic terms (in the $\psi$ fields) to the action we can solve for the constraint. The partition function can be rewritten to the form

$$
\begin{aligned}
\mathcal{Z} & =\frac{1}{2 \pi \mathrm{i}} \int_{-\mathrm{i} \infty}^{+\mathrm{i} \infty} \mathrm{e}^{-\lambda N} \mathrm{~d} \lambda \int\left[\mathcal{D} \psi \mathcal{D} \psi^{*}\right] \\
& \times \exp \left(-\sum_{i, j} \int_{0}^{\beta} \mathrm{d} \tau \mathrm{d} \tau^{\prime} \psi_{i}\left(\left(\tilde{t}_{b} I_{i j}+\lambda \delta_{i j}\right) \delta\left(\tau-\tau^{\prime}\right)\right.\right. \\
& \left.\left.\gamma_{i j}\left(\tau, \tau^{\prime}\right)\right) \psi_{j}^{*}\right)
\end{aligned}
$$

where

$$
\gamma_{i j}\left(\tau, \tau^{\prime}\right)=\left\langle\exp \left(-\mathrm{i}\left(\phi_{i}(\tau)-\phi_{j}\left(\tau^{\prime}\right)\right)\right)\right\rangle
$$

is the two-point phase correlator associated with the order parameter field, where $\langle\cdots\rangle$ denotes averaging with respect to the action in Eq. (12). Because the values of the phases $\phi$ which differ by $2 \pi$ are equivalent we decompose phase field in terms of a periodic field and term linear in $\tau$ : 


$$
\phi_{i}(\tau)=\varphi_{i}(\tau)+\frac{2 \pi}{\beta} m_{i} \tau,
$$

with $\phi_{i}(\beta)=\phi_{i}(0)$. As a result the phase correlator factorizes as the product of a topological term depending on the integers $m_{i}$ and non-topological one:

$$
\gamma_{i j}\left(\tau, \tau^{\prime}\right)=\gamma_{i j}^{T}\left(\tau, \tau^{\prime}\right) \gamma_{i j}^{N}\left(\tau, \tau^{\prime}\right) .
$$

Performing the Poisson re-summation formula in

$$
=\frac{\sum_{\left[m_{i}\right]}^{T} \mathrm{e}^{-\mathrm{i} \frac{2 \pi}{\beta}\left(\tau-\tau^{\prime}\right) m_{i}} \mathrm{e}^{-\frac{2 \pi}{\beta} \sum_{i}\left(\frac{\pi}{U_{\text {eff }}} m_{i}^{2}+\frac{\beta}{\mathrm{i}} \frac{\bar{\mu}_{b}}{U_{\text {eff }}} m_{i}\right)}}{\sum_{\left[m_{i}\right]} \mathrm{e}^{-\frac{2 \pi}{\beta} \sum_{i}\left(\frac{\pi}{U_{\text {eff }}} m_{i}^{2}+\frac{\beta}{\mathrm{i}} \frac{\bar{\mu}_{b}}{U_{\text {eff }}} m_{i}\right)}}
$$

and the functional integration over the phase variables

$$
\begin{aligned}
& \gamma_{i j}^{N}\left(\tau, \tau^{\prime}\right) \\
& =\frac{\int[\mathcal{D} \varphi] \mathrm{e}^{-\mathrm{i}\left(\varphi_{i}(\tau)-\varphi_{j}\left(\tau^{\prime}\right)\right)} \mathrm{e}^{-\sum_{i} \frac{1}{2 U_{\text {eff }}} \int_{0}^{\beta} \mathrm{d} \tau \dot{\varphi}_{i}^{2}(\tau)}}{\int[\mathcal{D} \varphi] \mathrm{e}^{-\sum_{i} \frac{1}{2 U_{\text {eff }}} \int_{0}^{\beta} \mathrm{d} \tau \dot{\varphi}_{i}^{2}(\tau)}}
\end{aligned}
$$

the final formula of the correlator takes the form

$$
\begin{gathered}
\gamma_{i j}\left(\tau, \tau^{\prime}\right)=\frac{\vartheta\left(\pi \frac{\bar{\mu}_{b}}{U_{\text {eff }}}+\pi \frac{\tau-\tau^{\prime}}{\beta}, \mathrm{e}^{-\frac{1}{U_{\text {eff }}} \frac{2 \pi^{2}}{\beta}}\right)}{\vartheta\left(\pi \frac{\bar{\mu}_{b}}{U_{\text {eff }}}, \mathrm{e}^{-\frac{1}{U_{\text {eff }}} \frac{2 \pi^{2}}{\beta}}\right)} \\
\times \exp \left(\frac{U_{\text {eff }}}{2}\left|\tau-\tau^{\prime}\right|-\frac{\left(\tau-\tau^{\prime}\right)^{2}}{\beta}\right),
\end{gathered}
$$

where $\vartheta(z, q)$ is the Jacobi theta function, which comes from the topological contribution - summation over integer winding numbers. The function $\vartheta(z, q)$ is defined by

$$
\vartheta(z, q)=1+2 \sum_{n=1}^{+\infty} \cos (2 n z) q^{n^{2}},
$$

and is $\beta$-periodic in the "imaginary time" as well in the variable $\bar{\mu}_{b} / U_{\text {eff }}$ with the period of unity which emphasizes the special role of its integer values. After Fourier transforming one obtains

$$
\begin{aligned}
& \gamma_{i j}\left(\omega_{\nu}\right) \\
& =\frac{1}{\mathcal{Z}_{0}} \frac{4}{U_{\text {eff }}} \sum_{\left[m_{i}\right]} \frac{\mathrm{e}^{-\frac{U_{\text {eff }} \beta}{2} \sum_{i}\left(m_{i}+\frac{\bar{\mu}_{b}}{U_{\text {eff }}}\right)^{2}}}{1-4\left(\sum_{i} m_{i}+\frac{\bar{\mu}_{b}}{U_{\text {eff }}}-\mathrm{i} \frac{\omega_{\ell}}{U_{\text {eff }}}\right)^{2}},
\end{aligned}
$$

where

$$
\mathcal{Z}_{0}=\sum_{\left[m_{i}\right]} \mathrm{e}^{-\frac{U_{\text {eff }} \beta}{2} \sum_{i}\left(m_{i}+\frac{\bar{\mu}_{b}}{U_{\text {eff }}}\right)^{2}},
$$

is the partition function for the set of quantum rotors. The action Eq. (12), with the topological contribution Eq. (24), after Fourier transform, is written as

$$
\mathcal{S}_{\text {eff }}[\psi, \bar{\psi}]=\frac{1}{N \beta} \sum_{\boldsymbol{k}, \ell} \bar{\psi}_{\boldsymbol{k}}\left(\omega_{\ell}\right) \Gamma_{\boldsymbol{k}}^{-1}\left(\omega_{\ell}\right) \psi_{\boldsymbol{k}}\left(\omega_{\ell}\right)
$$

where $\Gamma_{\boldsymbol{k}}^{-1}\left(\omega_{\ell}\right)=\lambda-t_{b \boldsymbol{k}}+\gamma^{-1}\left(\omega_{\ell}\right)$ is the inverse of the propagator and $t_{b \boldsymbol{k}}$ is the Fourier transform of the bosonic hopping matrix elements. Within the phase coherent state the order parameter $\psi_{B}$ is evaluated in the thermodynamic limit $N \rightarrow \infty$ by the saddle point method $\delta \mathcal{F} / \delta \lambda=0$ and the uni-modular condition of the $\mathrm{U}(1)$ phase variables translates into the equation

$$
1-\psi_{\mathrm{B}}^{2}=\lim _{N \rightarrow \infty} \frac{1}{N \beta} \sum_{\boldsymbol{k}, \ell} \Gamma_{\boldsymbol{k}}\left(\omega_{\ell}\right),
$$

with

$$
\begin{aligned}
& \Gamma_{\boldsymbol{k}}^{-1}\left(\omega_{\ell}\right)=\tilde{t}_{b \boldsymbol{k}=0}-\tilde{t}_{b \boldsymbol{k}}+\frac{1}{U_{\mathrm{eff}}} \bar{\mu}_{b}^{2} \\
& -\frac{1}{U_{\mathrm{eff}}}\left(\bar{\mu}_{b}-\mathrm{i} \omega_{\ell}\right)^{2} .
\end{aligned}
$$

The phase boundary is determined by the divergence of the order parameter susceptibility $\Gamma_{\boldsymbol{k}=0}\left(\omega_{\ell=0}\right)=0$

$$
\lambda_{0}-t_{b \boldsymbol{k}=0}^{\max }+\gamma^{-1}\left(\omega_{\ell=0}\right)=0,
$$

which determines the critical value of the Lagrange parameter $\lambda=\lambda_{0}$ and stays constant in the whole global coherent phase.

The critical line equation that separates the Mott insulator-superfluid transition, details of similar derivation of the critical line equation Eq. (27) are described in [11], will take simple form:

$$
\int_{-\infty}^{+\infty} \frac{\rho^{p / q}(\xi) \mathrm{d} \xi}{\sqrt{2 \bar{\xi}\left(8 \frac{t_{b}}{U_{b}}+\frac{\mu_{b}}{U_{b}}-\eta\right) \frac{1}{\alpha} \frac{t_{b}}{U_{b}}+v^{2}\left(\frac{1}{\alpha} \frac{\mu_{b}}{U_{b}}\right)}},
$$

where $\bar{\xi}=\xi_{\max }^{p / q}-\xi$ and $\xi_{\max }^{p / q}$ is the maximum of the band spectrum. The renormalization parameters are defined as:

$$
\alpha=1+\frac{U_{b f}^{2}}{U_{b}} \chi^{\prime}(\omega),
$$

and

$$
\eta=\frac{U_{b f}}{U_{b}} N_{\mathrm{F}}-1 / 2 .
$$

In Eq. (30) $v(\mu / U)=\operatorname{frac}(\mu / U)-1 / 2$, where $\operatorname{frac}(x)$ is the fractional part of the number. Because the higher values of the normalized chemical potential for the fermions $\mu_{f} / t_{f}$ decreases $\chi^{\prime}(\omega)$ and $\chi^{\prime \prime}(\omega)$ [11], consequently terms containing explicitly the average density of fermions $N_{\mathrm{F}}$ will acquire more significance than these with exclusively the inter-species interaction $U_{b f}$.

The periodicity of the phase diagram can be easily characterized by its evolution with changing the $\alpha$ parameter and applied magnetic field (see Figs. 2 and 3 ). The presence of fermions added to the system modifies the inter-species interaction and significantly changes the phase diagram causing both loss of the coherence or the stability of the superfluid phase. The resulting phase diagram and which phase the system reaches depends on the real part of the Lindhard function (Fig. 1). Experimentally we can measure absorption spectrum (imaginary part of the Lindhard function which maximum gives us precisely the resonant frequency position) and from that get the information whether we have reached the resonant regime or not. In conclusion, we have studied a mixture of bosons and spinless fermions confined in the two-dimensional square lattice with synthetic magnetic field imposed on the system. The strong effective mag- 


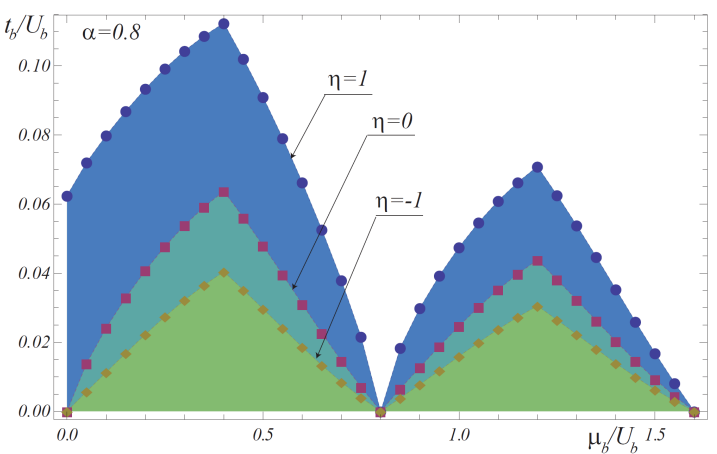

Fig. 2. Phase diagrams $\left(t_{b} / U_{b}-\mu_{b} / U_{b}\right)$ for the square $(2 D)$ lattice for $\alpha=1$ and different $\eta$. Dashed line stands for the phase boundary of one-component BoseHubbard model. Within the lobes the Mott insulator phase takes place with $\Psi_{\mathrm{B}}=0$.

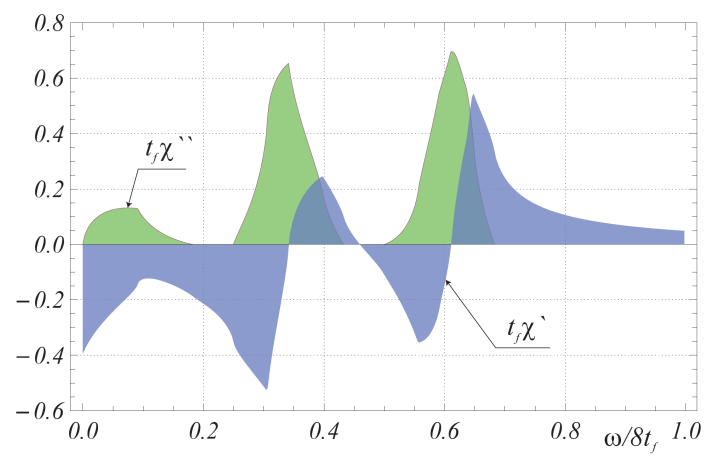

Fig. 3. Phase diagrams $\left(t_{b} / U_{b}-\mu_{b} / U_{b}\right)$ for the square $(2 D)$ lattice for $\alpha=0.8$ and different $\eta$. Dashed line stands for the phase boundary of one-component BoseHubbard model. Within the lobes the Mott insulator phase takes place with $\Psi_{\mathrm{B}}=0$.

netic field realizes the Berry's phase for a moving particles with different statistics. We found that reaching a quantum-Hall regime provides a very complex dynamics. Consequently the effective bosonic interaction to be switched between repulsive and attractive. The experimental evidence of our findings is feasible however precise measurements of the magnetic field are requisite which is possible with the recently developed optically synthesized magnetic field for neutral atoms [15] where Raman lasers applied to the gas realize a Berry's phase.

We thank T.K. Kopeć, R. Micnas and I. Spielman for discussion and comments regarding the paper.

\section{References}

[1] I. Coddington, P.C. Haljan, P. Engels, V. Schweikhard, S. Tung, E. A. Cornell, Phys. Rev. A 70, 063607 (2004).

[2] S. Tung, V. Schweikhard, E.A. Cornell, Phys. Rev. Lett. 97, 240402 (2006).

[3] V. Schweikhard, S. Tung, E. A. Cornell, Phys. Rev. Lett. 99, 030401 (2007).

[4] R. Bhat, M. Krämer, J. Cooper, M.J. Holland, Phys. Rev. A 76, 043601 (2007).

[5] C. Ospelkaus, S. Ospelkaus, K. Sengstock, K. Bongs, Phys. Rev. Lett. 96, 020401 (2006).

[6] F. Ferlaino, C. D'Errico, G. Roati, M. Zaccanti, M. Inguscio, G. Modugno, A. Simoni, Phys. Rev. A 73, 040702(R) (2006).

[7] K. Günter, T. Stöferle, H. Moritz, M. Köhl, T. Esslinger, Phys. Rev. Lett. 96, 180402 (2006).

[8] T. Best, S. Will, U. Schneider, L. Hackermüller, D. van Oosten, I. Bloch, Phys. Rev. Lett. 102, 030408 (2009).

[9] J. Catani, L. De Sarlo, G. Barontini, F. Minardi, M. Inguscio, Phys. Rev. A 77, 011603(R) (2008).

[10] R.M. Lutchyn, S. Tewari, S. Das Sarma, Phys. Rev. A 79, 011606(R) (2009).

[11] T.P. Polak, T.K. Kopeć, Phys. Rev. A 81, 043612 (2010).

[12] P. Buonsante, S.M. Giampaolo, F. Illuminati, V. Penna, A. Vezzani, Phys. Rev. Lett. 100, 240402 (2008).

[13] G. Mazzarella, Eur. Phys. J. D 50, 61 (2008).

[14] F. Illuminati, A. Albus, Phys. Rev. Lett. 93, 090406 (2004).

[15] Y.-J. Lin, R.L. Compton, K. Jimenez-García, J.V. Porto, I.B. Spielman, Nature 462, 628 (2009).

[16] S. Will, T. Best, U. Schneider, L. Hackermüller, D. Lühmann, I. Bloch, Nature 46, 197 (2010).

[17] A. Albus, F. Illuminati, J. Eisert, Phys. Rev. A 68, 023606 (2003).

[18] T.P. Polak, T.K. Kopeć, Phys. Rev. A 79, 063629 (2009).

[19] G. Giuliani, G. Vignale, Quantum Theory of the Electron Fluid, Cambridge 2005.

[20] S. Florens, A. Georges, Phys. Rev. B 70, 035114 (2004).

[21] T.K. Kopeć, Phys. Rev. B 70, 054518 (2004).

[22] E. Zhao, A. Paramekanti, Phys. Rev. B 76, 195101 (2007).

[23] T.P. Polak, T.K. Kopeć, Phys. Rev. B 76, 094503 (2007). 